\title{
(3-Aminopropyl)Triethoxysilane-Modified Silver Nanowire Network with Strong Adhesion to Coating Substrates for Highly Transparent Electrodes
}

\author{
Sangin Nam ${ }^{\dagger}$, Sung-Min Lee ${ }^{\dagger}$, Jin Kim, In-Hyeok Oh and Suk-Tai Chang * \\ School of Chemical Engineering and Materials Science, Chung-Ang University, 84 Heukseok-ro, Dongjak-gu, \\ Seoul 06974, Korea; sangin728@naver.com (S.N.); vision_2080@naver.com (S.-M.L.); rlawls0206@naver.com (J.K.); \\ ink409@naver.com (I.-H.O.) \\ * Correspondence: stchang@cau.ac.kr \\ + These authors contributed equally to this work.
}

check for updates

Citation: Nam, S.; Lee, S.-M.; Kim, J.; Oh, I.-H.; Chang, S.-T.

(3-Aminopropyl)Triethoxysilane-

Modified Silver Nanowire Network with Strong Adhesion to Coating Substrates for Highly Transparent Electrodes. Coatings 2021, 11, 499. https://doi.org/10.3390/ coatings11050499

Academic Editor: Alberto Palmero

Received: 2 April 2021

Accepted: 21 April 2021

Published: 23 April 2021

Publisher's Note: MDPI stays neutral with regard to jurisdictional claims in published maps and institutional affiliations.

Copyright: (c) 2021 by the authors. Licensee MDPI, Basel, Switzerland. This article is an open access article distributed under the terms and conditions of the Creative Commons Attribution (CC BY) license (https:// creativecommons.org/licenses/by/ $4.0 /)$.

\begin{abstract}
In this study, adhesion improved silver nanowires (AgNWs) conducting film was produced via the meniscus dragging deposition method. In order to improve adhesion of AgNWs films, the AgNWs were surface-modified with (3-aminopropyl)triethoxysilane (APTES) and coated over the pristine AgNWs networked film. Based on this strategy, the positively charged amine groups of the APTES-AgNWs and the negatively charged hydroxyl groups of the substrates formed electrostatic bonds, improving the adhesion between the AgNWs and substrates without sacrificing conductivity. AgNWs films on the rigid and flexible substrates were characterized using various analytical techniques. AgNWs networked film exhibited a sheet resistance of 6-22 $\Omega$ /sq at the transmittance at $550 \mathrm{~nm}$, corresponding to $74-86 \%$ transmittance, confirming promising transparent electrodes. Adhesion of AgNWs film is confirmed based on a peel-off test and AgNWs film maintained a good conductivity even after several peel-off tests.
\end{abstract}

Keywords: transparent electrode; silver nanowires; adhesion; thin films; solution process

\section{Introduction}

Transparent conducting electrodes (TCEs) are important components of optoelectronic devices, such as organic light emitting diodes, organic solar cells, and touch screens [1]. Owing to its excellent optical and electrical properties, indium tin oxide (ITO) is the most widely used material for TCEs [2]. However, the rigidity and rarity of ITO limit its application in flexible devices [3]. Overcoming these limitations is crucial for the advent of optoelectronic devices with innovative form factors. Hence, various materials such as metallic nanowires [4], carbon nanotubes [5], metallic grids [6], graphene sheets [7], and conductive polymers [8] have been investigated to replace ITO in flexible optoelectronic devices. Among them, Ag nanowires (AgNWs) have emerged as a promising alternative to ITO as they possess optoelectrical properties comparable to those of ITO as well as excellent mechanical flexibility. Moreover, they are cost-effective and can be solution-processed. Various solution-coating techniques such as spray coating [9-12], spin coating [13-16], drop coating [17], vacuum filtration/transfer [18], bar coating [19], Meyer rod coating [20-22], and the self-assembly method [23] have been used to prepare AgNWs. These facile and cost-effective solution processes have large-scale production efficiency comparable to that of ITO production methods.

Although AgNWs offer numerous advantages, they have several limitations such as poor adhesion to the coating substrate, high contact resistance at the wire/wire junction, surface roughness, and oxidation issues. Various approaches, including mechanical pressing [24,25], encapsulating AgNWs with other materials [26], and using adhesion promoters $[22,27,28]$, have been adopted to improve the adhesion of AgNWs or other nanomaterials to the coating substrate. 
The surface modification of substrates with silane groups is the most widely used approach to improve the adhesion of nanostructures onto them via electrostatic interactions. Recently, (3-aminopropyl)triethoxysilane (APTES) and (3-mercaptopropyl)trimethoxysilane (MPTES) have been used to functionalize the surfaces of various substrates with amine $(-\mathrm{NH} 2)$ and thiol $(-\mathrm{SH})$ groups, respectively. The silanol ( $\mathrm{Si}-\mathrm{OH})$ groups of APTES/MPTES interact with the hydroxyl $(-\mathrm{OH})$ groups of glass substrates pre-treated with piranha solution, while the amine/thiol groups interact with AgNWs via self-assembly of the wires [29]. However, the self-assembly method requires approximately 2-24 $\mathrm{h}$ for the fabrication of transparent films. To improve the performance of AgNW-based transparent conductive films, self-assembly is carried out via surface modification of the AgNWs and the substrate to produce a competitive figure of merit.

In this study, we fabricated thin films by depositing AgNWs on glass and polyester (PE) substrates. The AgNWs were surface-modified with APTES (APTES-AgNWs) to improve their adhesion to the substrates. The glass substrate was treated with piranha solution, while the PE substrate was treated with plasma to generate hydroxyl groups on their surface. The amine $\left(-\mathrm{NH}_{2}\right)$ group of the APTES-AgNWs interacted with the negatively charged hydroxyl $(-\mathrm{OH})$ group of the substrates, improving the adhesion between the substrate and AgNW films, minimizing sacrifice of transmittance and conductivity. The coating technique used in this study was meniscus dragging deposition (MDD), where a stacked film is formed by repeating the coating process, eventually reducing the time required for film formation. The substrates were first coated with bare AgNWs and then with APTES-AgNWs to improve the adhesion between them and the NWs, without significantly affecting the electrical conductivity and optical transparency of the resulting films. The rigid and flexible films were characterized using optical microscopy (OM), field emission scanning electron microscopy (FE-SEM), ultraviolet-visible-near-infrared (UV-Vis-NIR) spectroscopy, and the four-point probe method.

\section{Materials and Methods}

\subsection{Materials}

AgNW solution was purchased from DUKSAN Hi-Metal Co. Ltd., Ulsan, Korea. The AgNWs were dispersed in isopropyl alcohol (IPA) at a concentration of $0.5 \mathrm{wt} . \%$; the NWs had an average length of $40 \mu \mathrm{m}$ and a diameter of $40 \mathrm{~nm}$. APTES and IPA were purchased from Sigma-Aldrich and used to treat the surface of the AgNWs. To prepare the APTES-AgNWs, APTES was added to the AgNW solution at various volume ratios (AgNW solution:APTES = 5:1, 10:1, 50:1, 100:1, and 500:1). The resulting solution was vortex-mixed and left at room temperature for $12 \mathrm{~h}[30,31]$. The excess APTES in the IPA solution was removed via centrifuging. The APTES-AgNWs separated from the centrifuged mixture were dispersed again in IPA, and the solutions were diluted to various concentrations $(0.03$, $0.06,0.12,0.2,0.25$, and $0.32 \mathrm{wt} . \%$ ) by adding IPA.

\subsection{Film Fabrication}

Prior to the fabrication of the AgNW films, the surface of the glass slides $(25 \mathrm{~mm} \times 75 \mathrm{~mm}$ with plain ends, Fisher Scientific, Waltham, MA, USA) was hydrophilized and cleaned with piranha solution for $2 \mathrm{~h}$ and washed thoroughly with deionized (DI) water. Subsequently, the glass slides were dried with nitrogen gas to remove the piranha solution left on the surface. Polyester (PE) film was used as the flexible coating substrate. PE films (SKC, Seoul, Korea) were cleaned with IPA and dried with nitrogen gas. Following this, the dried PE films were subjected to an air plasma (PDC-32G, Harrick Plasma, Ithaca, NY, USA) treatment at $18 \mathrm{~W}$ for $5 \mathrm{~min}$ to render their surfaces hydrophilic via oxygenation. All the APTES-AgNWs films were fabricated using the MDD technique [32]. The deposition plate was placed on the surface of the coating substrate making a wedge. The AgNW solution was injected into the wedge between the coating substrate and the deposition plate and trapped using a 20- $\mu \mathrm{L}$ capillary. The deposition plate, which was connected to a motorized stage (AL1-1515-3S, Micro Motion Technology, Incheon, Korea), moved back and forth 
at a speed of $16.7 \mathrm{~mm} / \mathrm{s}$. The coating process was repeated up to three times (number of coatings, $\mathrm{NC}=1-3$ ). In the case of the upper layer in the multilayer films, $8 \mu \mathrm{L}$ of the APTES-AgNWs solution was injected into the wedge in the same way as mentioned above. The MDD coating process was repeated on the substrate coated with bare AgNWs at the same speed and $\mathrm{NC}=1$.

\subsection{Characterization}

The OM images of the films were obtained using an Olympus BX-51 optical microscope equipped with a high-resolution ProRes CF Scan digital CCD camera (Jenoptik, Jena, Germany). The FE-SEM images of the films were obtained using a Carl Zeiss SIGMA field emission scanning electron microscope at $5 \mathrm{kV}$ and a working distance of $5.1 \mathrm{~mm}$. The transmittance of the films was analyzed using UV-Vis-NIR spectroscopy (V-670, Jasco, Tokyo, Japan). The sheet resistance of the films was measured by employing the four-point probe method (Keithley 2400, Cleveland, OH, USA). Additionally, the adhesion stability of the films was evaluated using the scotch tape method. The tape (3M scotch filament tape) was pressed onto the films for $90 \mathrm{~s}$ and slowly peeled at an angle of $180^{\circ}$. This process was followed by ASTM standard adhesion measurements.

\section{Results and Discussion}

The schematic illustration of the fabrication process for the adhesion-enhanced AgNWs networked film is shown in Figure 1. The suspension of bare AgNWs dispersed in IPA was coated on the substrates using the MDD technique, producing uniform AgNWs networked films. Subsequently, the APTES-AgNWs were coated on the pre-deposited AgNW networked film in a similar manner. Owing to this strategy, the adhesion strength of the AgNW networked film was greatly improved, while maintaining excellent electrical conductivity and optical transmittance. Figure 2 shows the FE-SEM images of the bare AgNWs and APTES-AgNWs. It is evident from the figure that the APTES-AgNWs exhibit a thicker and rougher capping layer of APTES compared to bare AgNWs, which are capped by a polyvinylpyrrolidone (PVP) layer. This implies that the APTES molecules were sufficiently bound on the AgNW surface via covalent and hydrogen bonds [33].
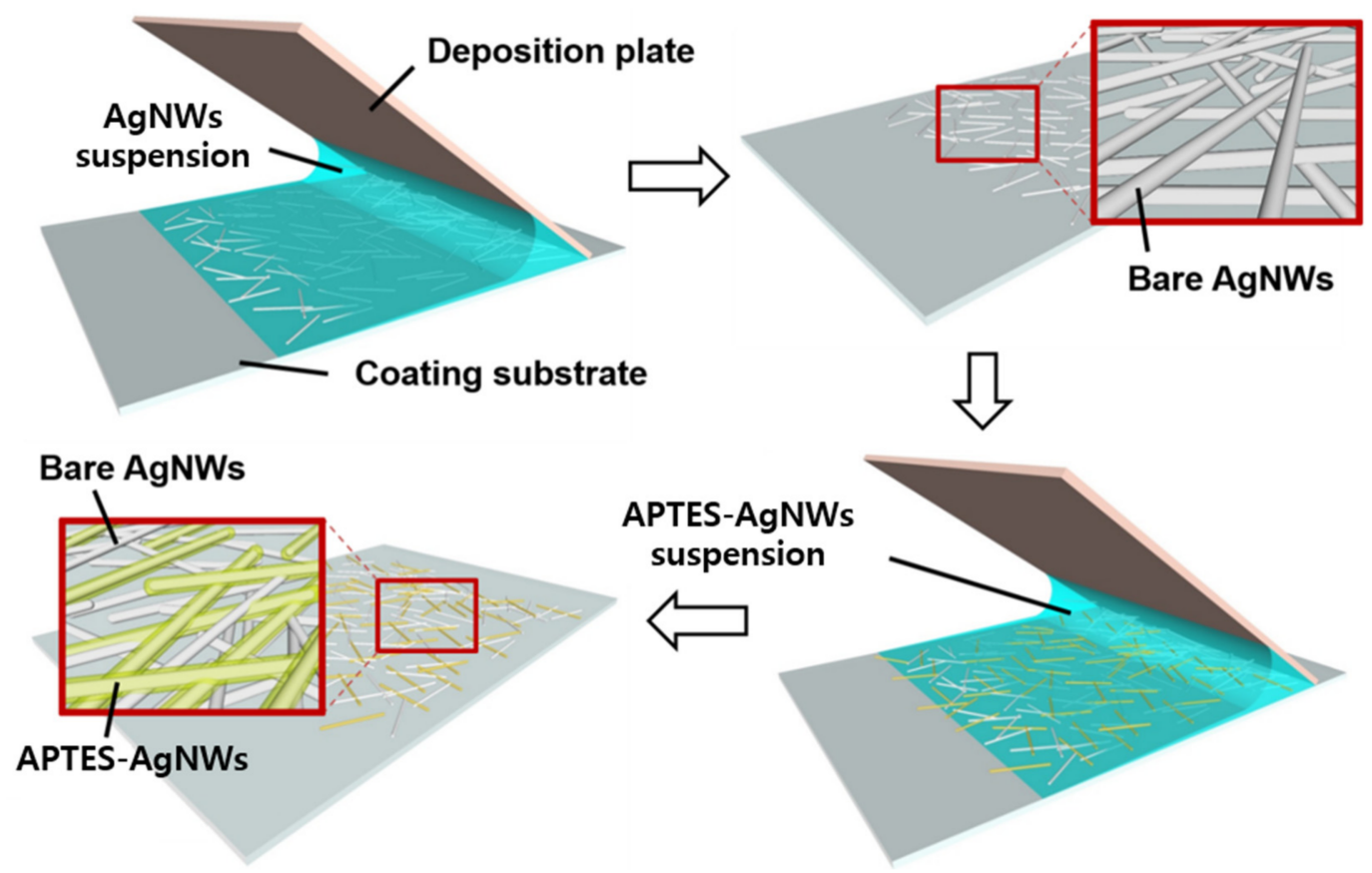

Figure 1. Schematic of the film deposition process using the MDD technique. 

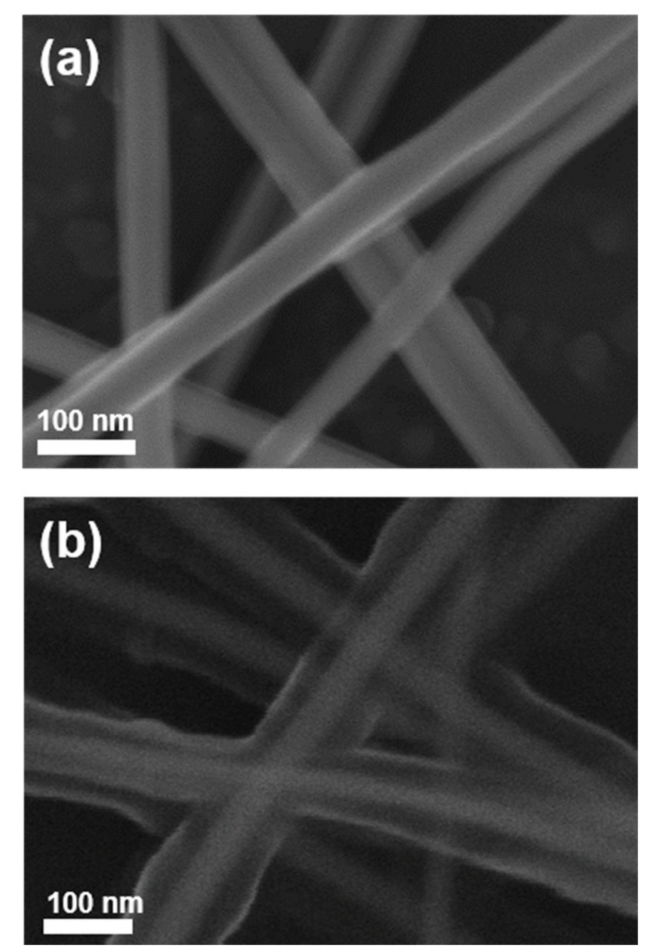

Figure 2. FE-SEM images of the (a) bare AgNWs and (b) APTES-AgNWs.

The principle of adhesion enhancement is explained in Figure 3. The APTES molecules comprise amine $\left(-\mathrm{NH}_{2}\right)$ functional groups and silanol groups $(\mathrm{Si}-\mathrm{OH})$, which have a high affinity to Ag and form covalent bonds with Ag atoms. The APTES molecules are randomly entangled with each other on the AgNWs via condensation and the formation of hydrogen bonds, forming a thick capping layer, as shown in Figure $2 \mathrm{~b}$. Owing to the random orientation of the APTES molecules in the capping layer, amine and silanol groups are abundant on the surface of the capping layer. Moreover, these functional groups can be strongly bound to the hydroxyl-rich glass slide and plasma-treated PE substrate, owing to the formation of hydrogen and siloxane bonds [34]. After annealing, the loosely bounded APTES molecuels are eliminated. Nevertheless, in this work, APTES-AgNWs networked film shows strong adhesion to the substrate after annealing because chemically bound APTES on the AgNWs surface are stable at $180{ }^{\circ} \mathrm{C}$ and it still contributes adhesion of APTES-AgNWs films [35]. To confirm the presence of the APTES capping layer, the N1s X-ray photoelectron spectroscopy (XPS) spectrum of the APTES-AgNWs was studied, as presented in Figure 3b. N1s spectrum of APTES-AgNWs was deconvoluted into two peaks at $402.2 \mathrm{eV}$ and $399.2 \mathrm{eV}$, corresponding to the amine groups from the APTES molecules and C-N bond from the PVP layer, respectively [30,31]. 
(a)
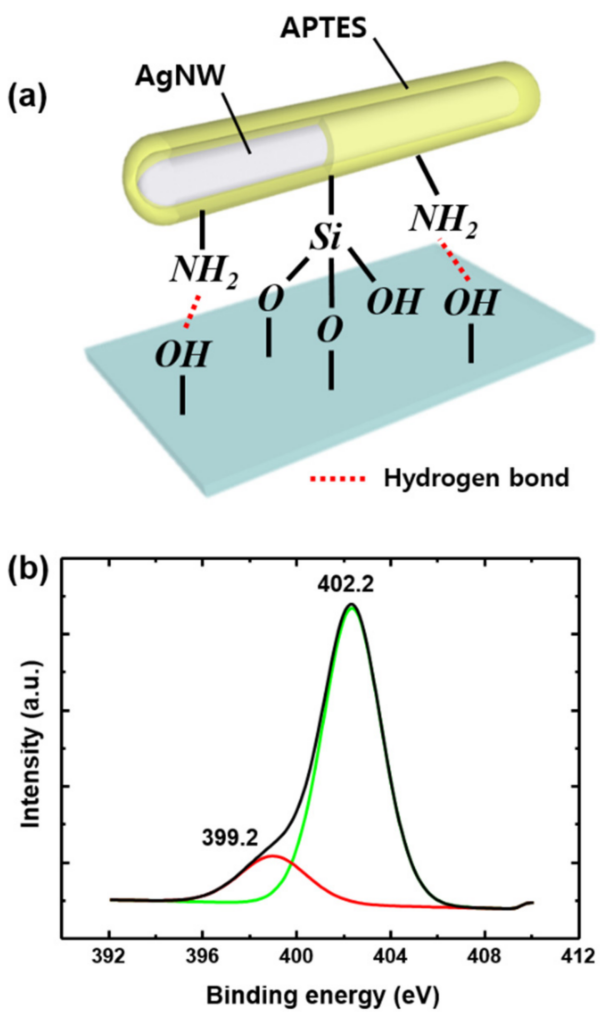

Figure 3. (a) Bonding between the APTES-AgNWs and glass substrate. (b) N1s XPS profiles of the APTES-AgNWs deposited on the glass substrate.

Further investigations involved the elucidation of the effect of the APTES concentration on the adhesion strength. The APTES treatment of AgNWs was carried out by adding APTES to the AgNW dispersion in various volume ratios (AgNWs solution:APTES = 500:1, 100:1, 50:1, 10:1, 5:1) at a fixed AgNW concentration of $0.5 \mathrm{wt} . \%$. Adhesion strength was evaluated by comparing transmittance at $550 \mathrm{~nm}$ before and after a peel-off test for APTESAgNWs networked films, as shown in Figure 4a and the result is summarized in Figure 4b. When the volume ratio of AgNWs solution:APTES is lower than 10:1, the transmittance of APTES-AgNWs networked film was greatly increased after the peel-off test, resulting from the significant delamination of APTES-AgNWs. In contrast, as the volume ratio of AgNWs solution:ATPES exceeds 10:1, the transmittance of the APTES-AgNWs film remains constant after the peel-off test, thus confirming the enhancement of adhesion by the APTES treatment. The corresponding FE-SEM images in Figure $4 \mathrm{c}$ and e illustrate the effect of the APTES treatment on the adhesion strength. At the AgNW solution:APTES volume ratio of 10:1, the surface of the AgNWs was sufficiently covered with APTES. However, at the AgNWs solution:APTES volume ratio of 100:1, the APTES exhibited an island-like growth on the surface of the AgNWs. Hence, the AgNWs were not sufficiently covered with the APTES. In addition, APTES was not observed on the surface of the AgNWs treated at the AgNW solution:APTES volume ratio of 500:1. 
(a)

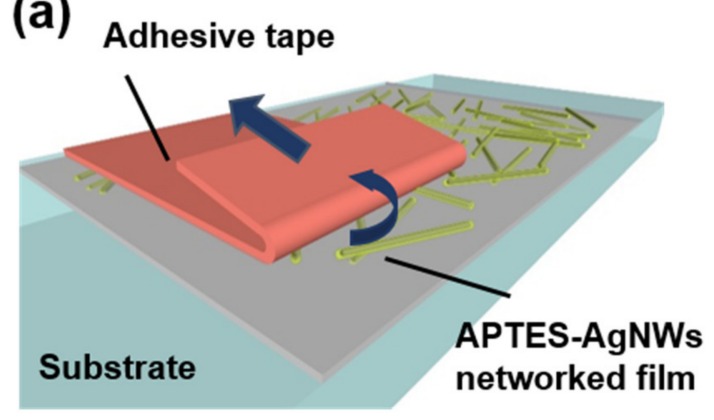

(b)

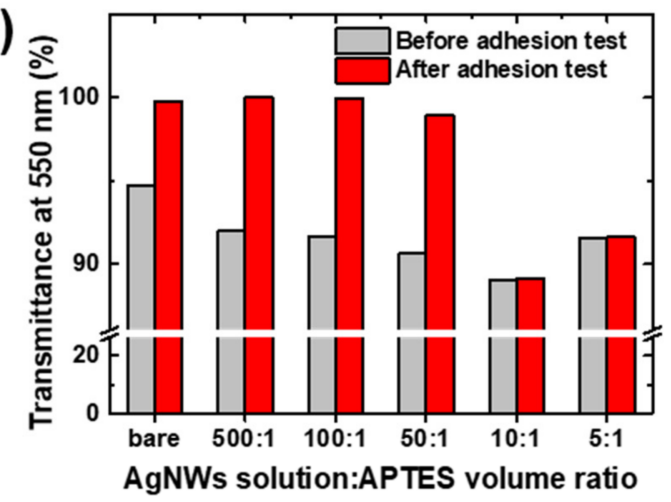

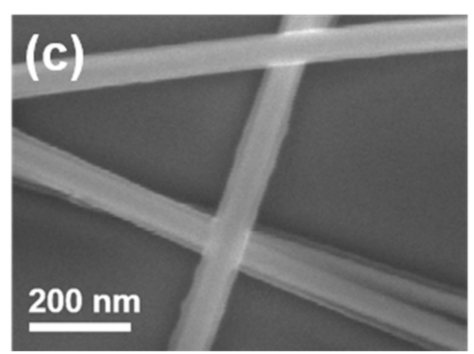
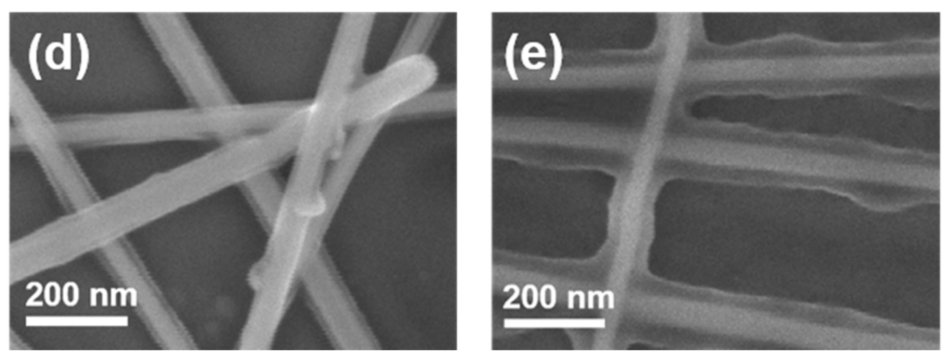

Figure 4. (a) Schematic illustration of the adhesion test for the APTES-AgNWs networked film. (b) Transmittance at $\lambda=550 \mathrm{~nm}$ of the APTES-AgNWs networked films on the glass substrate. The APTES-AgNWs were prepared by mixing 0.5 wt. $\%$ of the AgNW solution with APTES at the volume ratios of 500:1, 100:1, 50:1, 10:1, and 5:1. The APTES-AgNWs networked films were deposited directly on a glass substrate with DN $=3$ and NC $=1$. SEM images of the APTES-AgNWs with the AgNWs solution:APTES volume ratios of (c) 10:1, (d) 100:1, (e) 500:1.

The effect of the APTES-AgNWs solution concentration on the adhesion strength of APTES-AgNWs/AgNWs composite films was also investigated at a fixed APTES treatment condition (AgNW solution:APTES volume ratio $=10: 1$ ). As shown in Figure 5a, the bare AgNWs were coated on the substrate and the APTES-AgNWs were coated on the pre-deposited AgNWs networked film. Following this, the adhesion strength of the APTESAgNWs/AgNWs composite film was evaluated by comparing the transmittance of the composite film before and after the peel-off test. The concentration of APTES-AgNWs is varied between $0 \mathrm{wt} . \%$ and $0.32 \mathrm{wt} . \%$ and the results are summarized in Figure $5 \mathrm{~b}, \mathrm{c}$. It is evident from the results that before the peel-off test, the transmittance of the APTESAgNWs/AgNWs composite film only decreases slightly with increasing ATPES-AgNWs concentration. Meanwhile, the sheet resistance remains constant, thus indicating that ATPES-AgNWs do not contribute to the electrical conductivity and transmittance. However, after the peel-off test, the transmittance and sheet resistance are greatly increased at APTESAgNWs concentrations lower than $0.2 \%$. This is ascribed to the significant delamination of the APTES-AgNWs/AgNWs composite films. As Figure 5d shows, most AgNWs are delaminated after the peel-off test. In contrast, when the concentration of APTES-AgNWs is equal to or higher than $0.2 \%$, as Figure 5e shows, the APTES-AgNWs/AgNWs composite film is hardly delaminated after the peel-off test and the transmittance and sheet resistance remain unchanged, indicating the enhancement of adhesion strength. 
(a) Bare AgNWs

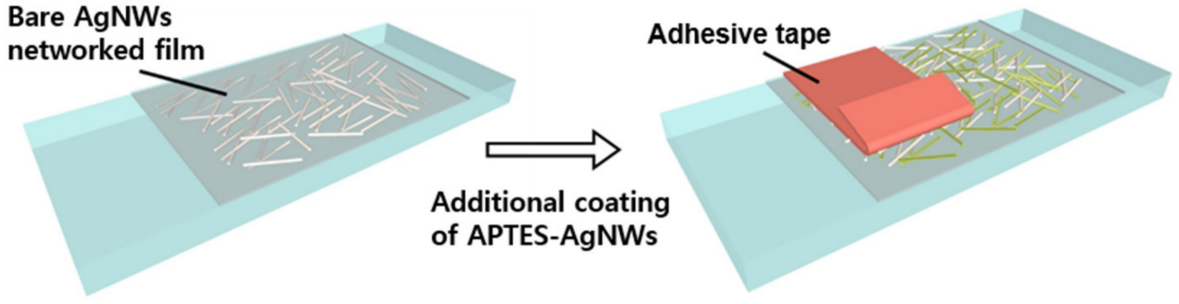

(b)

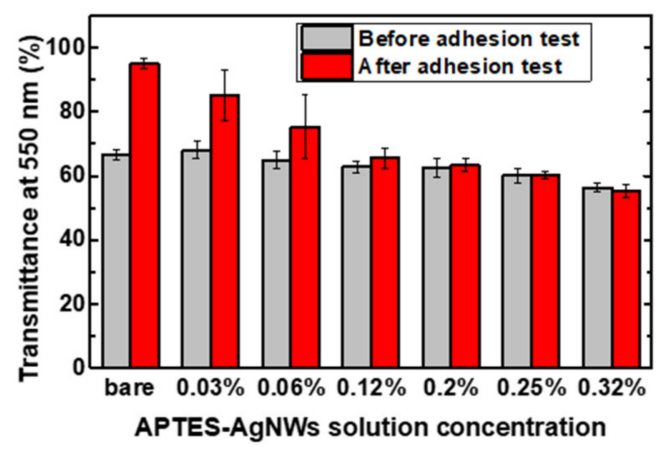

(d)

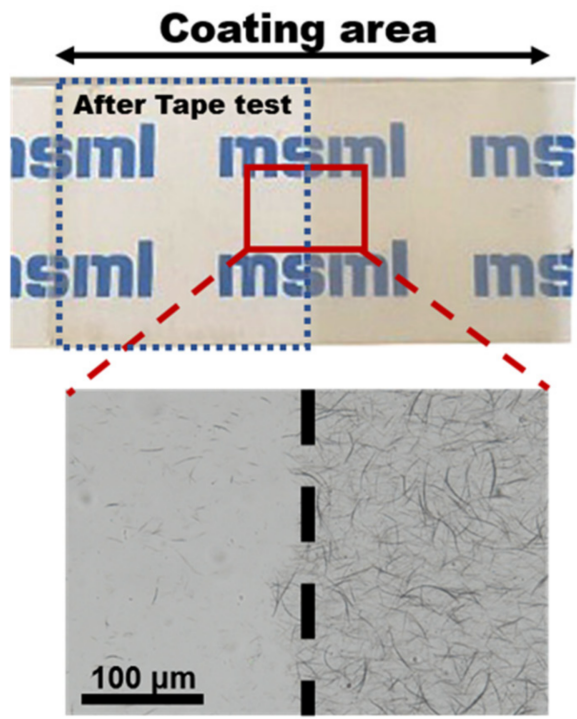

(c)

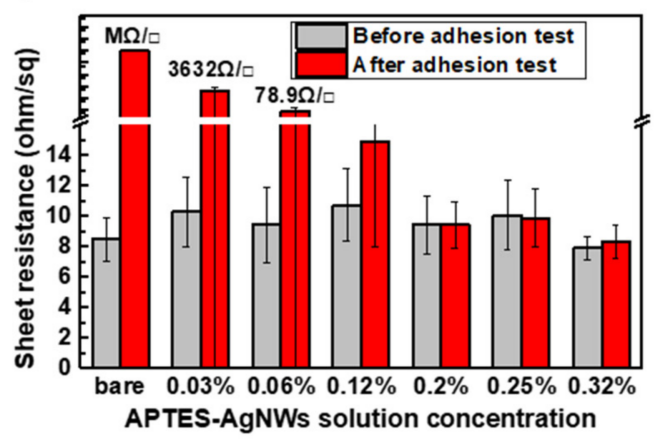

(e)

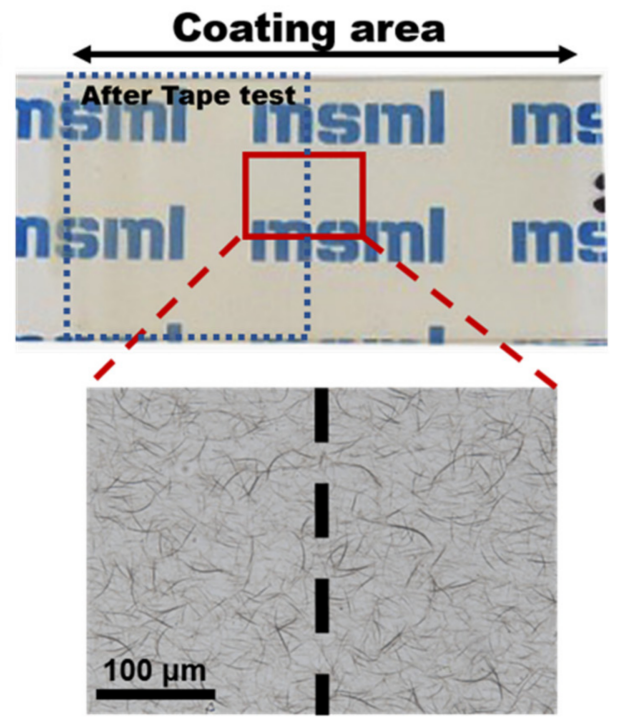

Figure 5. (a) Schematic of the preparation of the AgNWs thin films with the additional coating of the APTES-AgNWs and the tape test of the films. (b) Transmittance at $\lambda=550 \mathrm{~nm}$ and (c) sheet resistance of the AgNWs films with the APTES-AgNWs on a glass substrate as a function of the APTES-AgNW solution concentration $(0.03,0.06,0.12,0.2,0.25$, and 0.32 wt.\%). Photographs (top) and OM images (bottom) of (d) the bare AgNWs film and (e) APTES-AgNWs/AgNWs composite film before and after the tape test. The bare AgNWs films were deposited directly on the glass substrate using $20 \mu \mathrm{L}$ of the $0.5 \mathrm{wt} . \%$ bare AgNWs suspension at $\mathrm{DN}=3$ and $\mathrm{NC}=3$. The APTES-AgNWs were deposited on the bare AgNWs film using $8 \mu \mathrm{L}$ of the APTES-AgNWs suspension at $\mathrm{DN}=1$ and $\mathrm{NC}=1$.

The effect of the bare AgNWs deposition condition on the adhesion of APTESAgNWs/AgNWs composite films was investigated. The deposition amount of bare AgNWs was controlled by increasing the NC from 1 to 3, followed by the deposition of the APTES-AgNWs with NC $=1$. The result is summarized in Figure 6. The transmittance at $550 \mathrm{~nm}$ and the sheet resistance of the films decreased with increasing NC of the bare AgNWs, resulting from the more densely networked AgNWs layer. The adhesion test was repeated five times for each sample and no change was observed in the transmittance at $550 \mathrm{~nm}$, indicating that APTES-AgNWs/AgNWs composite films are appreciably adhered to the substrate. It is evident from Figure $6 \mathrm{~b}$ that the sheet resistance of the films decreased 
after the first peel-off test. It is attributed to the fact that the pressure applied during the peel-off test improved the contact in the junctions between the AgNWs, thus reducing the electrical resistance between the AgNWs [36]. Although there is little fluctuation of sheet resistance in the film with $\mathrm{NC}=1$ for bare AgNWs, the AgNWs thin films exhibit great adhesion to the glass slide even after the repetition of the peel-off test five times.

(a)

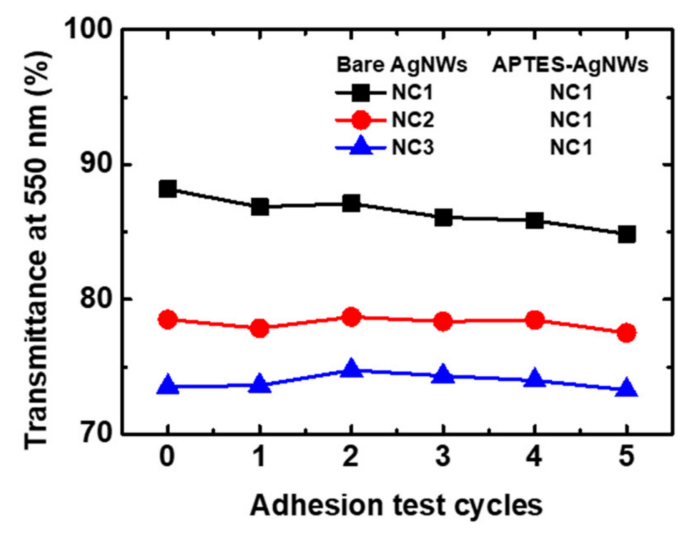

(b)

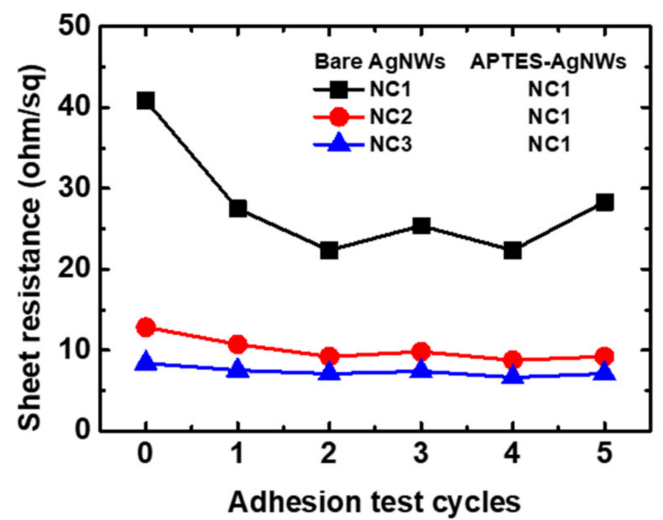

Figure 6. (a) Transmittance at $\lambda=550 \mathrm{~nm}$ and (b) sheet resistance of the APTES-AgNWs/AgNWs composite films as a function of the number of tape test cycles. The AgNW thin films were deposited on a glass substrate using $20 \mu \mathrm{L}$ of the $0.5 \mathrm{wt} . \%$ bare AgNW suspension at $\mathrm{NC}=1-3$. The APTESAgNWs were coated on the bare AgNWs deposited using $8 \mu \mathrm{L}$ of the $0.32 \mathrm{wt} . \%$ APTES-AgNW suspension at $\mathrm{NC}=1$.

Based on the above results, the electrical conductivity and transmittance of the APTESAgNWs/AgNWs composite film were finely tuned. The transmittance at $550 \mathrm{~nm}$ with respect to the sheet resistance was compared for the following three sample groups: (1) AgNWs networked films, (2) APTES-AgNWs/AgNWs composite films before the adhesion test, (3) APTES-AgNWs/AgNWs composite films after the adhesion test (Figure 7). The performance of the electrodes was analyzed based on the value of the figures of merit (FoM) calculated from the following equation:

$$
\mathrm{FoM}=\frac{\sigma_{D C}}{\sigma_{O P}}=\frac{188.5}{R_{S}\left(\frac{1}{\sqrt{T}}-1\right)}
$$

where $\sigma_{O P}$ is the optical conductivity and $\sigma_{D C}$ is the $D C$ conductivity of the metallic thin films. As is evident from Figure $7 \mathrm{a}$, the transmittance and sheet resistance values of the APTES-AgNWs/AgNWs composite film are similar to those of the AgNWs networked film without an APTES-AgNWs layer under a wide range of sheet resistance values. This indicates that the adhesion strength is effectively improved, thus minimizing the loss of conductivity and transmittance. The highest FoM values for each group were calculated 
to be 152.4 for AgNWs and 135.0 and 174.4 for APTES-AgNWs/AgNWs composite films before and after the adhesion test, respectively. As previously discussed, the increase in FoM after the adhesion test is a result of the welding effect arising from the applied pressure during the adhesion test. The electrical conductivity of the AgNW-based electrodes can be increased using thermal annealing. The annealing was carried out $180^{\circ} \mathrm{C}$ for $5 \mathrm{~min}$, the results of which are summarized in Figure $7 \mathrm{~b}$. The highest FoM values of the annealed bare AgNWs films, APTES-AgNWs/AgNWs composite film before adhesion test, and APTES-AgNWs/AgNWs composite films after the adhesion test are calculated to be 201, 177 , and 195, respectively. This increase of FoM after annealing is ascribed to the melting of the PVP capping layer of the AgNWs surface, thus reducing the resistance of the junctions between the wires [32].

\section{(a)}

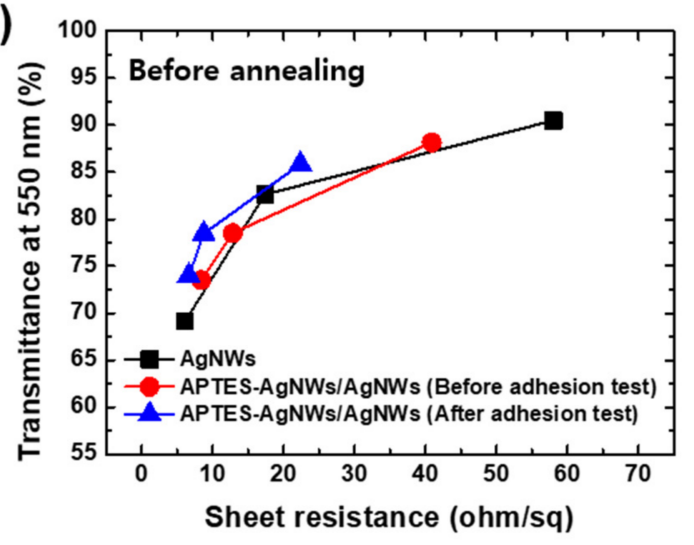

(b)

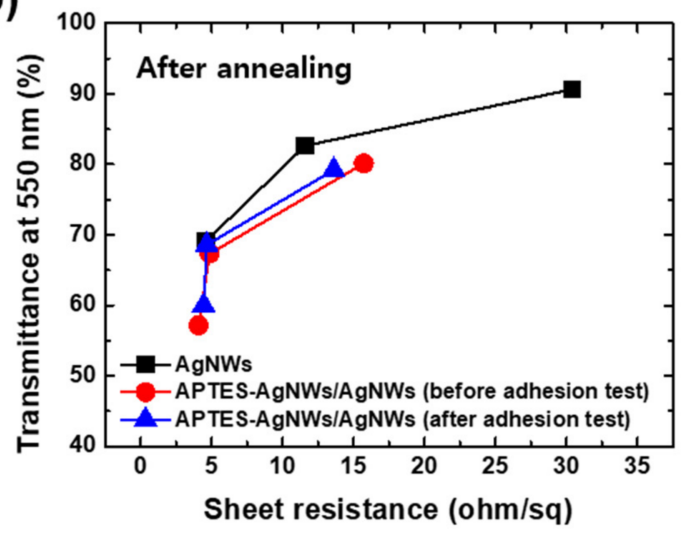

Figure 7. Transmittance at $\lambda=550 \mathrm{~nm}$ of the AgNWs networked films (a) before and (b) after annealing as a function of the sheet resistance. The solid lines are the fit to Equation (1). The bare AgNWs films were deposited on the glass substrate using $20 \mu \mathrm{L}$ of the $0.5 \mathrm{wt}$. $\%$ AgNWs suspension at NC $=1-3$. The APTES-AgNWs were coated on the bare AgNWs networked films using $8 \mu \mathrm{L}$ of the 0.32 wt. $\%$ APTES-AgNW suspension at $\mathrm{NC}=1$. The AgNWs films were annealed at $180{ }^{\circ} \mathrm{C}$ for $5 \mathrm{~min}$ on a hotplate.

The statistical percolation theory was employed to further investigate the optical and electrical properties of the AgNWs networked films. Figure 8a,b show the surface resistance of the AgNWs-based films with and without annealing with respect to the surface concentration. As AgNWs networked films exhibit a linear relation with the amount of the AgNWs deposited on the substrate, the surface concentration of the AgNWs can be calculated from light absorbance. According to the percolation theory, the sheet resistance and surface concentration of a transparent conductor exhibit the following relation:

$$
R_{S} \propto\left(x-x_{c}\right)^{-\alpha}
$$


where $R_{S}$ is the surface resistance, $x$ is surface concentration, $x_{c}$ is the percolation threshold, and $\alpha$ is the percolation exponent [37]. The fitted line of Equation (2) is also shown in the graphs. The $x_{c}$ values of the bare AgNWs films, APTES-AgNWs / AgNWs composite films, and APTES-AgNWs/AgNWs composite films after the peel-off test (Figure 8a) were calculated to be $2.81,3.48$, and $2.32 \mu \mathrm{g} / \mathrm{cm}^{2}$, respectively. The $x_{c}$ values of the annealed bare AgNW films, APTES-AgNWs/AgNWs composite films, and APTES-AgNWs/AgNWs composite films after the peel-off test were $2.12,2.85$, and $2.14 \mu \mathrm{g} / \mathrm{cm}^{2}$, respectively (Figure $8 \mathrm{~b}$ ). The smaller $x_{c}$ values indicate that the electrical connections between each of the AgNWs are improved after annealing, resulting in an increase in conductivity. The $\alpha$ value, which indicates the dimensionality of the films, is close to 1.33 (the values of the $\alpha$ varied from 1.01 to 1.53 in this study), corresponding to the value of the two-dimensional conduction model cited in the literature. Overall, the trend of sheet resistance with respect to the surface concentration corresponds well with Equation (2) for all samples. This indicates the fabrication of well-structured AgNWs networked with wide-ranged controllability.
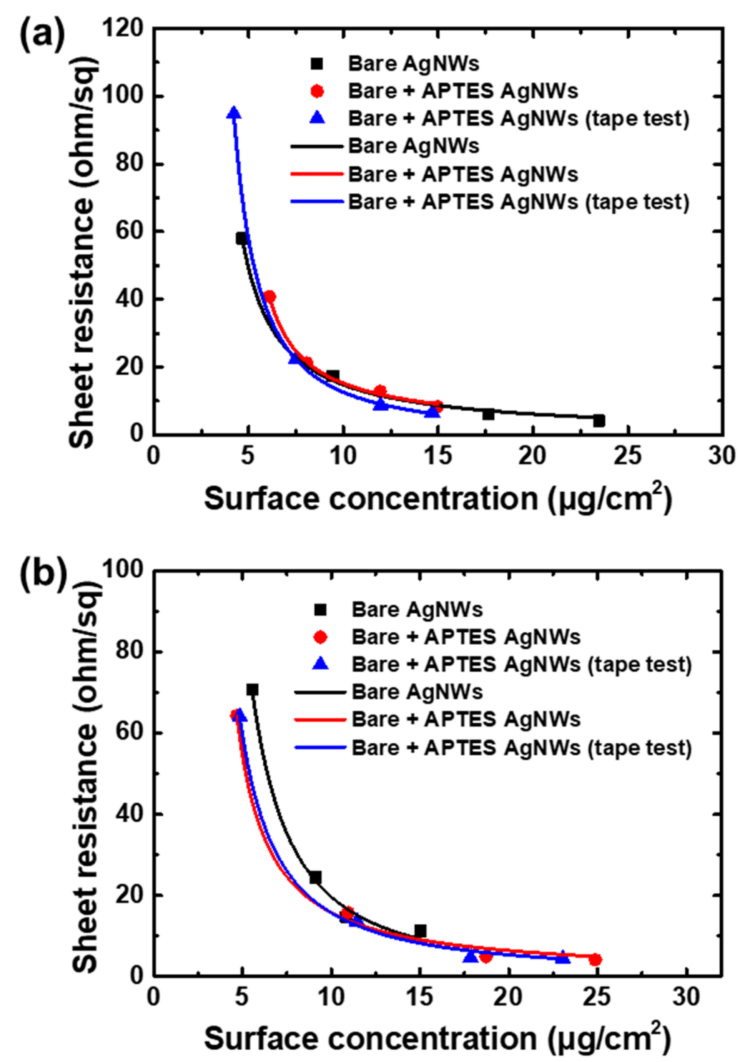

Figure 8. Sheet resistance of the AgNWs networked thin films as a function of the AgNWs surface concentration (a) before annealing and (b) after annealing. The solid lines are a fit to the percolation equation (Equation (2)).

To confirm the feasibility of the fabricated APTES-AgNWs/AgNWs composite films as flexible electrodes, we fabricated APTES-AgNWs/AgNWs composite films on a PE substrate, as shown in Figure 9a. Firstly, the bending stability of the electrodes was investigated, and the change of resistance over repeated bending cycles was measured. As shown in Figure 9b, the resistance hardly changed, even after 2500 bending cycles. Moreover, the bending characteristic curve for the APTES-AgNWs/AgNWs composite films was also investigated under compressive and tensile loading. Figure $9 \mathrm{c}, \mathrm{d}$ exhibit the relative resistance $\left(R / R_{0}\right)$ of the films as a function of the bending radius $(\mathrm{mm})$, where $R$ is 
the resistance measured under stress and $R_{0}$ is the resistance without stress. The bending radius $\left(R_{\text {nom }}\right)$ of the films was calculated using the following equation:

$$
R_{\text {nom }}=\frac{L}{2 \pi \sqrt{d L / L-\pi^{2} h_{s}^{2} / 12 L^{2}}}
$$

where $L, d L / L$, and $h_{S}$ denote the initial length, applied strain, and thickness of the substrate, respectively $[38,39]$. As shown in Figure $9 c, d$, the films exhibited excellent flexibility and maintained their electrical conductivity under compressive and tensile loading, even when the bending radius was decreased to approximately $2 \mathrm{~mm}$.

(a)

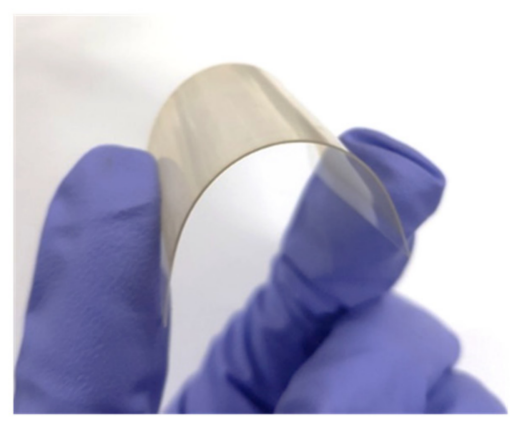

(c)

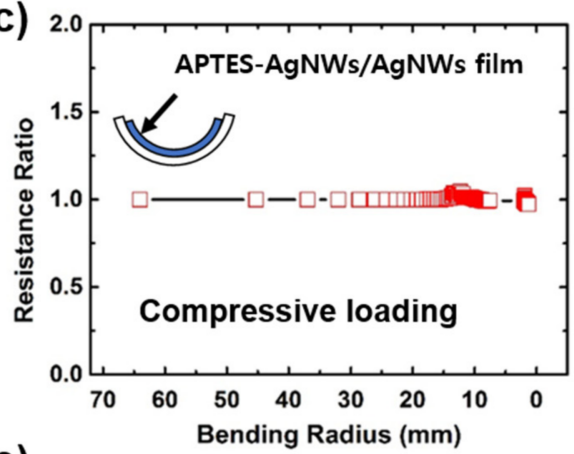

(e)

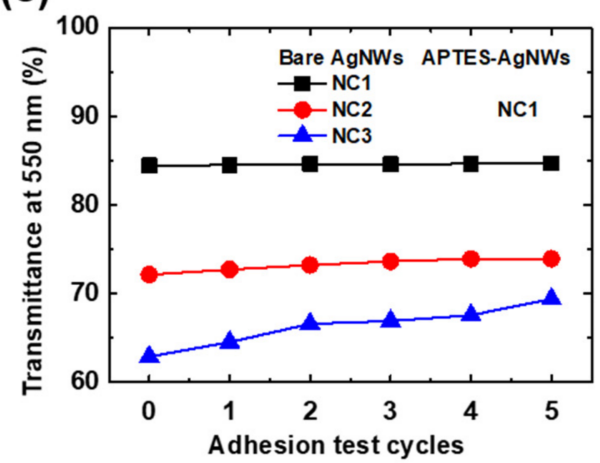

(b)

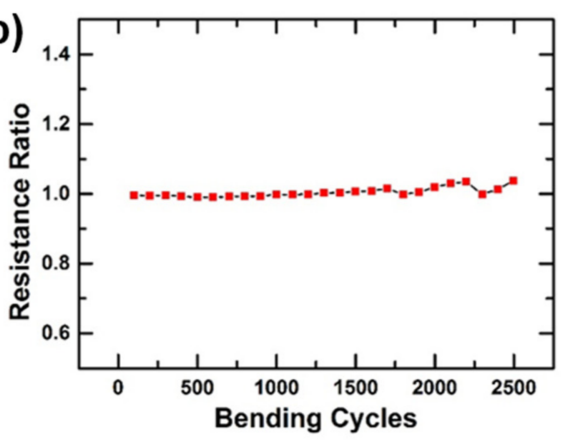

(d)

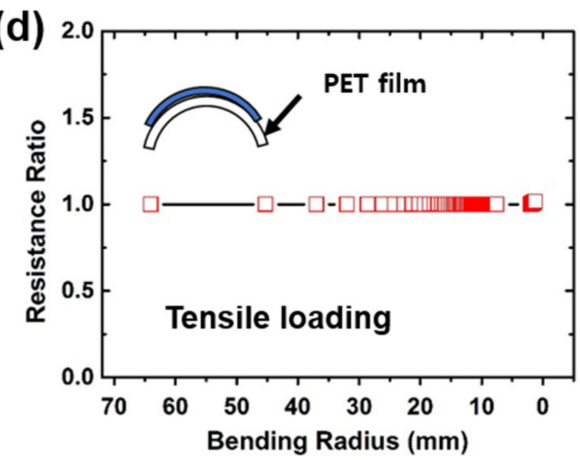

(f)

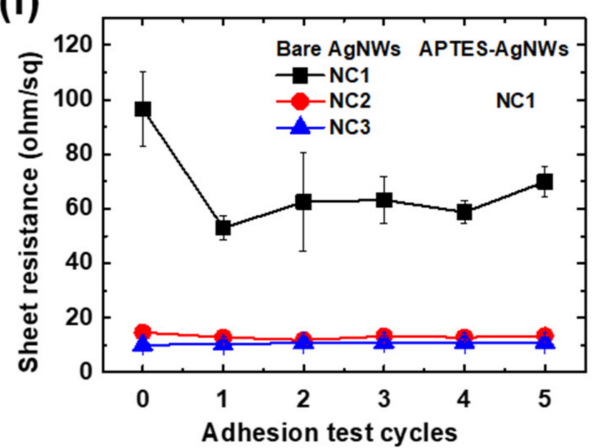

Figure 9. (a) Photograph of the APTES-AgNWs/AgNWs composite film deposited on a PE substrate. (b) Relative sheet resistance of the APTES-AgNWs/AgNWs composite film deposited on a PE substrate as a function of the number of compressive bending cycles. Relative sheet resistance of the APTES-AgNWs / AgNWs composite film with respect to the bending radius on a PE substrate as under the (c) compressive and (d) tensile loading. (e) Transmittance at $\lambda=550 \mathrm{~nm}$ and (f) sheet resistance of the APTES-AgNWs/AgNWs composite films as a function of the number of tape test cycles. The bare AgNWs networked films were deposited on the PE substrate using $20 \mu \mathrm{L}$ of the 0.5 wt. $\%$ AgNWs solution at NC $=1-3$. The APTES-AgNWs were deposited on the bare AgNWs using $8 \mu \mathrm{L}$ of the $0.32 \mathrm{wt}$.\% APTES-AgNW suspension at $\mathrm{NC}=1$. 
The adhesion test was employed to investigate the adhesion strength between the APTES-AgNWs/AgNWs composite and the PE substrate (Figure 9e,f). First, the bare AgNWs were coated on the plasma-treated PE substrate. Following this, the APTESAgNWs were coated on the bare AgNWs and the peel-off test was carried out. The NC of the bare AgNWs was varied from 1 to 3. It is evident from Figure 9e that when the NC of the bare AgNWs was 1 and 2, the transmittance of the films did not change over the course of the test cycle. However, at $\mathrm{NC}=3$, the transparency of the films increased slightly with the repetition of the peel-off tests. This indicates that the adhesion between the APTES-AgNW layer and the substrate was poor because of the presence of a thick, bare AgNW layer. However, the $\mathrm{NC}=3$ sample maintained its sheet resistance even after multiple rounds of the peel-off test (Figure 9f). This was attributed to the delamination arising from the excess amounts of APTES-AgNWs, which do not contribute to the conductivity. The amine $\left(-\mathrm{NH}_{2}\right)$ groups present in the APTES layer formed electrostatic bonds with the hydroxylated PE substrate, resulting in the strong adhesion between the APTES-AgNWs / AgNWs composite film and PE substrate [40]. Thus, flexible transparent conductive films with good adhesion were successfully fabricated by coating the APTES-AgNWs on the bare AgNWs.

\section{Conclusions}

We fabricated AgNWs thin films strongly adhered on rigid (glass slide) and flexible (PE) substrates using the MDD coating technique. The thin films can be employed as transparent electrodes as alternatives to ITO. The MDD technique enabled the fine tuning of the transmittance and sheet resistance values of the electrodes by controlling the coating parameters, such as the AgNW solution concentration, and the NC of the bare AgNWs injection volume [32]. The APTES-AgNWs layer improved the adhesion of the AgNWs networked film to the substrates without loss of transmittance and sheet resistance. The adhesive strength was enhanced by the electrostatic bonds between the hydroxyl (-OH) group on the surface of the substrate the amine $\left(-\mathrm{NH}_{2}\right)$ and silanol $(\mathrm{Si}-\mathrm{OH})$ groups on the surface of the APTES-AgNWs. The surface modification of AgNWs with APTES is a facile and cost-effective process and promotes adhesion to the substrate, thus minimizing performance degradation. Therefore, we expect our work to contribute to the wide-spread application of AgNW-based electrodes in the field of flexible electronics.

Author Contributions: Conceptualization, S.N., S.-M.L., and S.-T.C.; methodology, S.N. and S.-M.L.; validation, S.N., S.-M.L., J.K., and S.-T.C.; investigation, S.N., S.-M.L., J.K., and I.-H.O.; resources, S.N., S.-M.L., and S.-T.C.; data curation, S.N., S.-M.L., J.K., I.-H.O., and S.-T.C.; writing-original draft preparation, S.N. and S.-M.L.; writing-review and editing, S.N., S.-M.L., J.K., I.-H.O., and S.-T.C.; supervision, S.-T.C.; project administration, S.-T.C.; funding acquisition, S.-T.C. All authors have read and agreed to the published version of the manuscript.

Funding: This work was supported by the National Research Foundation of Korea (NRF) grant funded by the Korean government (MSIT) (No. 2019R1A2C1006413) and the Competency Development Program for Industry Specialists of the Koran Ministry of Trade, Industry and Energy (MOTIE) operated by Korea Institute for Advancement of Technology (KIAT) (No. P0012453, Next-generation Display Expert Training Project for Innovation Process and Equipment, Materials Engineers).

Institutional Review Board Statement: Not applicable.

Informed Consent Statement: Not applicable.

Data Availability Statement: Data is contained within the article.

Conflicts of Interest: The authors declare no conflict of interest. 


\section{References}

1. Morales-Masis, M.; De Wolf, S.; Woods-Robinson, R.; Ager, J.W.; Ballif, C. Transparent electrodes for efficient optoelectronics. Adv. Electron. Mater. 2017, 3, 1600529. [CrossRef]

2. Gicevicius, M.; Celiesiute, R.; Kucinski, J.; Ramanaviciene, A.; Bagdziunas, G.; Ramanavicius, A. Analytical Evaluation of Optical pH-Sensitivity of Polyaniline Layer Electrochemically Deposited on ITO Electrode. J. Electrochem. Soc. 2018, 165, H903-H907. [CrossRef]

3. Cairns, D.R.; Witte, R.P.; Sparacin, D.K.; Sachsman, S.M.; Paine, D.C.; Crawford, G.P.; Newton, R.R. Strain-dependent electrical resistance of tin-doped indium oxide on polymer substrates. Appl. Phys. Lett. 2000, 76, 1425-1427. [CrossRef]

4. Liu, S.; Chen, Z.; Zhang, N.; Tang, Z.R.; Xu, Y.J. An efficient self-assembly of CdS nanowires-reduced graphene oxide nanocomposites for selective reduction of nitro organics under visible light irradiation. J. Phys. Chem. C 2013, 117, 8251-8261. [CrossRef]

5. Dai, H. Carbon nanotubes: Opportunities and challenges. Surf. Sci. 2002, 500, 218-241. [CrossRef]

6. Jang, S.; Jung, W.-B.; Kim, C.; Won, P.; Lee, S.G.; Cho, K.M.; Jin, M.L.; An, C.J.; Jeon, H.J.; Ko, S.H.; et al. A three-dimensional metal grid mesh as a practical alternative to ITO. Nanoscale 2016, 8, 14257-14263. [CrossRef] [PubMed]

7. Sun, J.; Chen, Z.; Yuan, L.; Chen, Y.; Ning, J.; Liu, S.; Ma, D.; Song, X.; Priydarshi, M.K.; Bachmatiuk, A.; et al. Direct chemicalvapor-deposition-fabricated, large-scale graphene glass with high carrier mobility and uniformity for touch panel applications. ACS Nano 2016, 10, 11136-11144. [CrossRef]

8. Abdelhamid, M.E.; O'Mullane, A.P.; Snook, G.A. Storing energy in plastics: A review on conducting polymers \& their role in electrochemical energy storage. RSC Adv. 2015, 5, 11611-11626. [CrossRef]

9. Jin, Y.; Deng, D.; Cheng, Y.; Kong, L.; Xiao, F. Annealing-free and strongly adhesive silver nanowire networks with long-term reliability by introduction of a nonconductive and biocompatible polymer binder. Nanoscale 2014, 6, 4812-4818. [CrossRef] [PubMed]

10. Nengduo, Z.; Xuesong, Y.; Hao, G. Highly conductive and flexible transparent films based on silver nanowire/chitosan composite. RSC Adv. 2016, 6, 47552-47561. [CrossRef]

11. Hauger, T.C.; Al-Rafia, S.M.I.; Buriak, J.M. Rolling silver nanowire electrodes: Simultaneously addressing adhesion, roughness, and conductivity. ACS Appl. Mater. Interfaces 2013, 5, 12663-12671. [CrossRef]

12. Kang, H.; Kim, Y.; Cheon, S.; Yi, G.R.; Cho, J.H. Halide Welding for Silver Nanowire Network Electrode. ACS Appl. Mater. Interfaces 2017, 9, 30779-30785. [CrossRef] [PubMed]

13. Liu, R.; Tan, M.; Zhang, X.; Xu, L.; Chen, J.; Chen, Y.; Tang, X.; Wan, L. Solution-processed composite electrodes composed of silver nanowires and aluminum-doped zinc oxide nanoparticles for thin-film solar cells applications. Sol. Energy Mater. Sol. Cells 2018, 174, 584-592. [CrossRef]

14. Wang, T.; Luo, C.; Liu, F.; Li, L.; Zhang, X.; Li, Y.; Han, E.; Fu, Y.; Jiao, Y. Highly transparent, conductive, and bendable Ag nanowire electrodes with enhanced mechanical stability based on polyelectrolyte adhesive layer. Langmuir 2017, 33, 4702-4708. [CrossRef]

15. Lian, L.; Dong, D.; Feng, D.; He, G. Low roughness silver nanowire flexible transparent electrode by low temperature solutionprocessing for organic light emitting diodes. Org. Electron. 2017, 49, 9-18. [CrossRef]

16. Chung, C.H.; Song, T.B.; Bob, B.; Zhu, R.; Yang, Y. Solution-processed flexible transparent conductors composed of silver nanowire networks embedded in indium tin oxide nanoparticle matrices. Nano Res. 2012, 5, 805-814. [CrossRef]

17. Wang, J.; Jiu, J.; Sugahara, T.; Nagao, S.; Nogi, M.; Koga, H.; He, P.; Suganuma, K.; Uchida, H. Highly reliable silver nanowire transparent electrode employing selectively patterned barrier shaped by self-masked photolithography. ACS Appl. Mater. Interfaces 2015, 7, 23297-23304. [CrossRef]

18. Zeng, X.Y.; Zhang, Q.K.; Yu, R.M.; Lu, C.Z. A new transparent conductor: Silver nanowire film buried at the surface of a transparent polymer. Adv. Mater. 2010, 22, 4484-4488. [CrossRef]

19. Hoeng, F.; Denneulin, A.; Krosnicki, G.; Bras, J. Positive impact of cellulose nanofibrils on silver nanowire coatings for transparent conductive films. J. Mater. Chem. C 2016, 4, 10945-10954. [CrossRef]

20. Hu, L.; Kim, H.S.; Lee, J.Y.; Peumans, P.; Cui, Y. Scalable coating and properties of transparent, flexible, silver nanowire electrodes. ACS Nano 2010, 4, 2955-2963. [CrossRef] [PubMed]

21. Liu, G.S.; Qiu, J.S.; Xu, D.H.; Zhou, X.; Zhong, D.; Shieh, H.P.D.; Yang, B.R. Fabrication of embedded silver nanowires on arbitrary substrates with enhanced stability via chemisorbed alkanethiolate. ACS Appl. Mater. Interfaces 2017, 9, 15130-15138. [CrossRef]

22. Sun, Q.; Lee, S.J.; Kang, H.; Gim, Y.; Park, H.S.; Cho, J.H. Positively-charged reduced graphene oxide as an adhesion promoter for preparing a highly-stable silver nanowire film. Nanoscale 2015, 7, 6798-6804. [CrossRef]

23. Liu, S.; Weng, B.; Tang, Z.R.; Xu, Y.J. Constructing one-dimensional silver nanowire-doped reduced graphene oxide integrated with CdS nanowire network hybrid structures toward artificial photosynthesis. Nanoscale 2015, 7, 861-866. [CrossRef]

24. Kang, H.S.; Choi, J.; Cho, W.; Lee, H.; Lee, D.; Lee, D.G.; Kim, H.T. Silver nanowire networks embedded in a cure-controlled optical adhesive film for a transparent and highly conductive electrode. J. Mater. Chem. C 2016, 4, 9834-9840. [CrossRef]

25. Lian, L.; Dong, D.; Yang, S.; Wei, B.; He, G. Highly conductive and uniform alginate/silver nanowire composite transparent electrode by room temperature solution processing for organic light emitting diode. ACS Appl. Mater. Interfaces 2017, 9, 11811-11818. [CrossRef]

26. Jin, Y.; Li, L.; Cheng, Y.; Kong, L.; Pei, Q.; Xiao, F. Cohesively enhanced conductivity and adhesion of flexible silver nanowire networks by biocompatible polymer sol-gel transition. Adv. Funct. Mater. 2015, 25, 1581-1587. [CrossRef] 
27. Nonahal, M.; Rastin, H.; Saeb, M.R.; Sari, M.G.; Moghadam, M.H.; Zarrintaj, P.; Ramezanzadeh, B. Epoxy/PAMAM dendrimermodified graphene oxide nanocomposite coatings: Nonisothermal cure kinetics study. Prog. Org. Coat. 2018, 114, $233-243$. [CrossRef]

28. Abdi, A.A.; Jouyandeh, M.; Vahabi, H.; Shabanian, M.; Lafon-Pham, D.; Gabrion, X.; Laheurte, P.; Nahavandi, A.M.; Saeb, M.R. Correlating the Photophysical Properties with the Cure Index of Epoxy Nanocomposite Coatings. J. Inorg. Organomet. Polym. Mater. 2021, 31, 923-933. [CrossRef]

29. Tugba Camic, B.; Shin, H.J.; Hasan Aslan, M.; Basarir, F.; Choi, H. Solution-processable transparent conducting electrodes via the self-assembly of silver nanowires for organic photovoltaic devices. J. Colloid Interface Sci. 2018, 512, 158-164. [CrossRef]

30. Min, H.; Girard-Lauriault, P.L.; Gross, T.; Lippitz, A.; Dietrich, P.; Unger, W.E.S. Ambient-ageing processes in amine self-assembled monolayers on microarray slides as studied by ToF-SIMS with principal component analysis, XPS, and NEXAFS spectroscopy. Anal. Bioanal. Chem. 2012, 403, 613-623. [CrossRef] [PubMed]

31. Liang, Z.; Graham, K.R. Surface Modification of Silver Nanowires for Morphology and Processing Control in Composite Transparent Electrodes. ACS Appl. Mater. Interfaces 2015, 7, 21652-21656. [CrossRef] [PubMed]

32. Ko, Y.; Song, S.K.; Kim, N.H.; Chang, S.T. Highly transparent and stretchable conductors based on a directional arrangement of silver nanowires by a microliter-scale solution process. Langmuir 2016, 32, 366-373. [CrossRef]

33. Argekar, S.U.; Kirley, T.L.; Schaefer, D.W. Determination of structure-property relationships for 3-aminopropyltriethoxysilane films using X-ray reflectivity. J. Mater. Res. 2013, 28, 1118-1128. [CrossRef]

34. Kim, J.; Seidler, P.; Wan, L.S.; Fill, C. Formation, structure, and reactivity of amino-terminated organic films on silicon substrates. J. Colloid Interface Sci. 2009, 329, 114-119. [CrossRef]

35. Nabil, B.; Morshed, M.N.; Ahmida, E.A.; Nemeshwaree, B.; Christine, C.; Julien, V.; Olivier, T.; Abdelkrim, A. Development of new multifunctional filter based nonwovens for organics pollutants reduction and detoxification: High catalytic and antibacterial activities. Chem. Eng. J. 2019, 356, 702-716. [CrossRef]

36. Lee, S.J.; Kim, Y.H.; Kim, J.K.; Baik, H.; Park, J.H.; Lee, J.; Nam, J.; Park, J.H.; Lee, T.W.; Yi, G.R.; et al. A roll-to-roll welding process for planarized silver nanowire electrodes. Nanoscale 2014, 6, 11828-11834. [CrossRef]

37. Madaria, A.R.; Kumar, A.; Ishikawa, F.N.; Zhou, C. Uniform, highly conductive, and patterned transparent films of a percolating silver nanowire network on rigid and flexible substrates using a dry transfer technique. Nano Res. 2010, 3, 564-573. [CrossRef]

38. Park, S.I.; Ahn, J.H.; Feng, X.; Wang, S.; Huang, Y.; Rogers, J.A. Theoretical and experimental studies of bending of inorganic electronic materials on plastic substrates. Adv. Funct. Mater. 2008, 18, 2673-2684. [CrossRef]

39. Lim, J.W.; Cho, D.Y.; Eun, K.; Choa, S.H.; Na, S.I.; Kim, J.; Kim, H.K. Mechanical integrity of flexible Ag nanowire network electrodes coated on colorless PI substrates for flexible organic solar cells. Sol. Energy Mater. Sol. Cells 2012, 105, 69-76. [CrossRef]

40. Tamai, T.; Watanabe, M.; Kobayashi, Y.; Nakahara, Y.; Yajima, S. Surface modification of PEN and PET substrates by plasma treatment and layer-by-layer assembly of polyelectrolyte multilayer thin films and their application in electroless deposition. RSC Adv. 2017, 7, 3155-33161. [CrossRef] 